\title{
Oblique shock waves in granular flows over bluff bodies
}

\author{
Nandu Gopan ${ }^{1, \star}$ and Meheboob Alam ${ }^{1}$ \\ ${ }^{1}$ Jawaharlal Nehru Centre for Advanced Scientific Research, Jakkur PO, Bangalore 560064, INDIA
}

\begin{abstract}
Granular flows around an object have been the focus of numerous analytical, experimental and simulation studies. The structure and nature of the oblique shock wave developed when a quasi-two dimensional flow of spherical granular particles streams past a fixed cylindrical obstacle forms the focus of this study. The binary granular mixture, consisting of particles of the same diameter but different material properties, is investigated by using a modified LIGGGHTS package as the simulation engine. Variations of the solid fraction and granular temperature are analysed and the shock-front is identified. The local Mach number is calculated to distinguish between the subsonic and the supersonic regions of the bow shock.
\end{abstract}

\section{Introduction}

The out of equilibrium nature of granular matter leads to dynamically complex flows. The flow of granular matter around an immersed object is a subject of considerable interest given the wide range of scenarios where such configurations are found- a better understanding of the flow dynamics would be essential not just to design better equipment for granular particle processing and handling but also to better grasp the dynamics of flows where thermal agitation plays no role.

Granular flows around an object have been the focus of numerous analytical [1-3], experimental $[1,2]$ and simulation studies $[1,4]$.The formation of plane shock waves [5], when a granular medium is perturbed faster than the velocity of sound, has been reported by previous studies. Experimental studies [6, 7] have shown that a stagnant zone formation, similar to the classical "bow-shock" detachment (see Fig. 1), is possible in granular flows. Analytical relations [5] for granular shocks have been developed and Discrete Element Modelling (DEM) simulation studies $[4,8]$ of single component granular flow past obstacles have been reported in literature.

The present study analyses results from soft particle DEM simulations of two component granular flows over a bluff body. The structure and nature of the oblique shock wave developed when a quasi-two dimensional flow of spherical granular particles streams past an immersed, fixed cylindrical obstacle forms the focus of this study. The aim is to analyse the bow-shock structure in a binary granular mixture and contrast it with well-known predictions for related molecular gas flows.

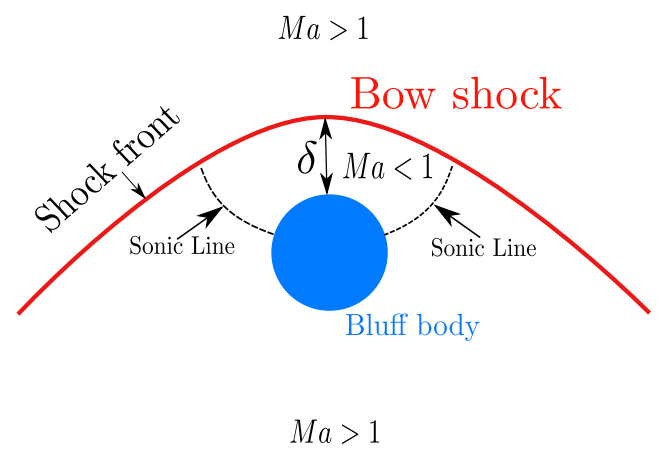

Figure 1: Schematic of bow-shock structure in molecular gas flow over a bluff body. The sonic line demarcates the subsonic and the supersonic regions, while $\delta$ denotes the detachment length.

Table 1: Simulation details

\begin{tabular}{ll}
\hline Simulation Parameter & Value \\
\hline Particle diameter, $d(\mathrm{~m})$ & 0.001 \\
Time-step (s), $\Delta t$ & $5 \times 10^{-7}$ \\
Upstream solid fraction, $\phi_{\infty}$ & 0.1 \\
Obstacle diameter, $D$ & $10 d / 50 d / 100 d$ \\
Upstream Velocity, $U_{\infty}\left(\frac{m}{s}\right)$ & $0.475 / 1 / 2.375$ \\
Simulation domain size, $x \times y$ & $500 d \times 500 d$ \\
Upstream Mach Number, $M a_{\infty}$ & $2.64 / 5.9 / 13.7$ \\
\hline
\end{tabular}

\section{Configuration and Numerical Method}

The flow configuration is shown in Fig. 2 and the simulation details are given in Table 1.The DEM simulations are carried out by employing a modified version of the opensource software LIGGGHTS [9]. The granular flow consists of a binary mixture of steel and aluminium particles which flow over a cylindrical shaped obstacle, of length

\footnotetext{
^e-mail: nandug@jncasr.ac.in
} 


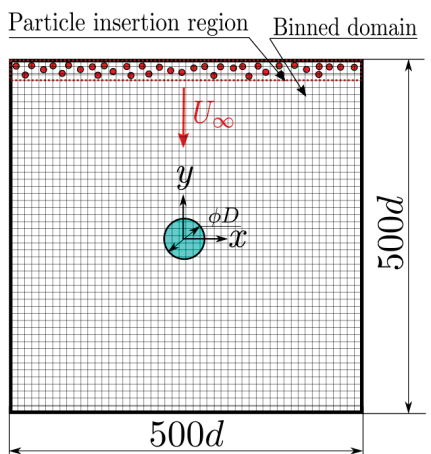

Figure 2: Schematic of the simulation domain. $D$ and $d$ denote the diameters of the cylindrical obstacle and granular particle, respectively, while $x$ and $y$ represent the crosswise and streamwise directions, respectively. The particle insertion region spans $2 d$ along the streamwise direction and $500 \mathrm{~d}$ along the crosswise direction. The simulation domain is binned into square regions of side $3 d$ for the calculation of macroscopic fields.

Table 2: Hertzian contact force model

\begin{tabular}{cc}
\hline Coefficient & Particle expression \\
\hline$\beta$ & $\frac{\ln (e)}{\sqrt{\ln (e)^{2}+\pi^{2}}}$ \\
$k_{n}^{*}=\frac{k_{n}}{\sqrt{\delta_{n}}}$ & $\frac{E \sqrt{d}}{3\left(1-\nu^{2}\right)}$ \\
$k_{t}^{*}=\frac{k_{t}}{\sqrt{\delta_{n}}}$ & $3 \frac{1-v}{2-v} k_{n}^{*}$ \\
$\gamma_{n}^{*}=\frac{\gamma_{n}}{\sqrt[4]{\delta_{n}}}$ & $\beta \sqrt{\frac{5 m_{e f f} k_{n}^{*}}{2}}$ \\
$\gamma_{t}^{*}=\frac{\gamma_{t}}{\sqrt[4]{\delta_{t}}}$ & $\beta \sqrt{\frac{5 m_{e f f} k_{t}^{*}}{3}}$ \\
\hline
\end{tabular}

$d$, modelled as aluminium. The Young's modulus $(E)$ of steel and aluminium are taken as $210 \mathrm{GPa}$ and $69 \mathrm{GPa}$, respectively, while the corresponding Poisson's ratios $(v)$ are taken to be 0.303 and 0.33 ; the material density of steel is taken as $\rho_{\text {Steel }}=7850 \mathrm{~kg} / \mathrm{m}^{3}$, with $\rho_{\text {Steel }} / \rho_{A l}=2.907$; the effective mass is $m_{e f f}=m_{\text {Steel }} m_{A l} /\left(m_{\text {Steel }}+m_{A l}\right)$. The coefficient of restitution $(e)$ of all particles is set to 0.5 . The particles have equal contribution to the upstream solid fraction of 0.1 , representing an equimolar binary mixture.

The normal and tangential forces between particles are calculated, respectively, as:

$$
\begin{gathered}
F_{n}=k_{n} \delta_{n}+\gamma_{n} v_{n}^{r e l} \\
F_{t}= \begin{cases}k_{t} \delta_{t}+\gamma_{t} v_{t}^{r e l}, & \text { if }\left|F_{t}\right| \leq \mu_{f}\left|F_{n}\right| \\
\mu_{f}\left|F_{n}\right|, & \text { otherwise }\end{cases}
\end{gathered}
$$

Here, $\mu_{f}$ is the coefficient of Coulomb friction while subscripts $n$ and $t$ denote the normal and tangential components, respectively. The parameters $k_{n}, k_{t}, \gamma_{n}$ and $\gamma_{t}$ are calculated from the Young's modulus $(E)$, Poisson's ratio $(v)$ and the coefficient of restitution (e) as given in Table 2. For all results presented in this study, $\mu_{f}$ is set to zero.

The total simulation time consists of 5 seconds of real time, of which the production run lasts for 4 seconds. The positions, velocities and the forces on each particle are noted and the macroscopic properties of interest are calculated by post-processing the particle trajectory data using an in-house post-processing code; the simulation domain is divided into a number of square bins of side $3 d$ (see Fig. 2) and the macroscopic fields (density, granular temperature, Mach number, etc) in each bin are calculated by averaging over the entire production run.

In each bin of volume $V_{b i n}$, the number-density of species $\alpha$ (steel or aluminium) is calculated from $n_{\alpha}=$ $N_{\alpha}^{b i n} / V_{b i n}$ where $N_{\alpha}^{b i n}$ is the 'average' number of particles of type $\alpha$ in that bin (averaged over time), and the local mass-density of species $\alpha$ is defined as

$$
\varrho_{\alpha}=m_{\alpha} n_{\alpha} \equiv \rho_{\alpha} \phi_{\alpha}
$$

where $\rho_{\alpha}$ is the "material" density of species $\alpha$ and $\phi_{\alpha}$ is its volume fraction in a particular bin. The mixture/total mass-density is $\varrho=\sum_{\alpha} \varrho_{\alpha}=\varrho_{S t e e l}+\varrho_{A l}$. The mean velocity of each species is calculated from $\mathbf{u}_{\alpha}=\left\langle c_{\alpha}\right\rangle$, where $\mathbf{c}_{\alpha}$ is the instantaneous velocity of species $\alpha$, with the angular bracket denoting averaging over time. The "mixture" velocity is defined as the mass-averaged velocity $\mathbf{u}=\sum_{\alpha} \varrho_{\alpha} \mathbf{u}_{\alpha} / \varrho$. The "species" granular temperature is calculated from

$$
T_{\alpha}=\frac{1}{\mathcal{D}}\left\langle m_{\alpha}\left(\mathbf{c}_{\alpha}-\mathbf{u}\right)^{2}\right\rangle,
$$

and the "mixture" granular temperature is $T=\sum_{\alpha} \xi_{\alpha} T_{\alpha}$, where $\xi_{\alpha}=n_{\alpha} / n$ is the "local" (binwise) number fraction of species $\alpha$.

Given the dilute nature of the flows considered, the flow regime can be understood to be inertial. The upstream Mach number, $M a_{\infty}$ is calculated via

$$
M a_{\infty}=\frac{U_{\infty}}{a_{\infty}}
$$

where the adiabatic sound speed, $a$, is given by [10]:

$$
a=\sqrt{\left(\frac{\partial P}{\partial \rho}\right)_{s}}=\left[\frac{T}{m}\left(1+\frac{2}{\mathcal{D}} F(\phi)+\frac{\phi}{F} \frac{d F}{d \phi}\right) F\right]^{1 / 2}
$$

where $\mathcal{D}$ is the dimensionality of the system and

$$
F(\phi)=1+(\mathcal{D}-1)(1+e) \phi g(\phi), \quad m=\sum_{\alpha} m_{\alpha}
$$

and $g(\phi)$ is the radial distribution function

$$
g(\phi)=\frac{16-7 \phi}{16(1-\phi)^{2}} .
$$

Since the granular temperature varies along the streamwise direction, we have calculated the upstream sound-speed $a_{\infty}$ by using $T$ and $\phi$ averaged over two-bins from the inlet.

\section{Results and Discussion}

The simulations with (i) three different obstacle diameters $(D=10 d, 50 d$ and $100 d)$ and (ii) three upstream Mach numbers $\left(M_{\infty}=2.64,5.9\right.$ and 13.7) have been analysed. Note that the upstream Mach number $M a_{\infty}$ has been varied by varying the upstream inflow velocity $U_{\infty}$ (see Table 1 ). 
(a)

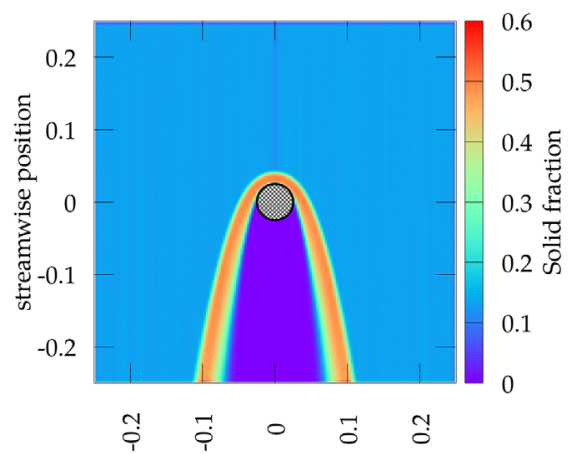

crosswise position

(b)

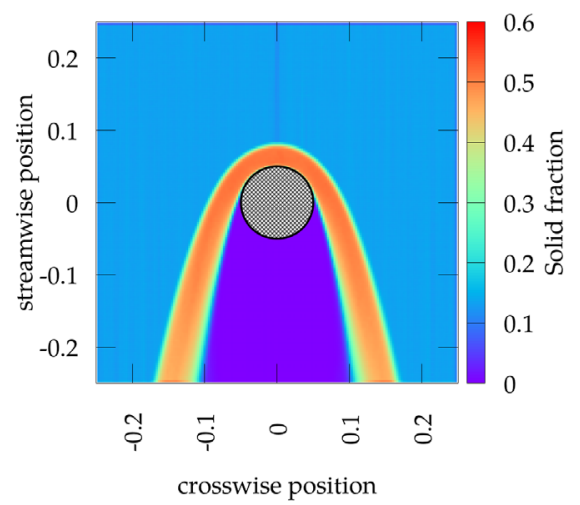

Figure 3: Solid fraction $(\phi)$ field calculated by binaveraging over the entire production run, for (a) $D=50 \mathrm{~d}$ and (b) $D=100 d$. Both cases are for $M a_{\infty}=5.9$.

\subsection{Identification of Shock Front}

The visualisation of particle trajectories at the end of the production run clearly shows a region of higher density around the cylindrical obstacle, reminiscent of a "bow shock", which is evident from the color-map of solid fraction in Fig. 3. Interestingly, the structure of the bow shock formed is qualitatively different from that known of a molecular gas (viz. Fig. 1) such that the region behind the obstacle (along the streamwise direction) is almost devoid of particles (Fig. 3) in the case of granular flows.

Analysing the dimensionless temperature field in Fig. 4, we find that a region of high temperature occurs between the shock wave front and the cylindrical obstacle. This seems counter-intuitive given the fact that one would expect the solid fraction and the granular temperature to be inversely dependent. However, this relationship is valid only for dilute granular flows. As the solid fraction fields (Fig. 3) have revealed, the solid fraction in the region between the shock front and the cylindrical obstacle is nearly 0.5 , which cannot be considered dilute. This calls for an analysis of the stress-field behind the shock front, which can be taken up in future. (a)
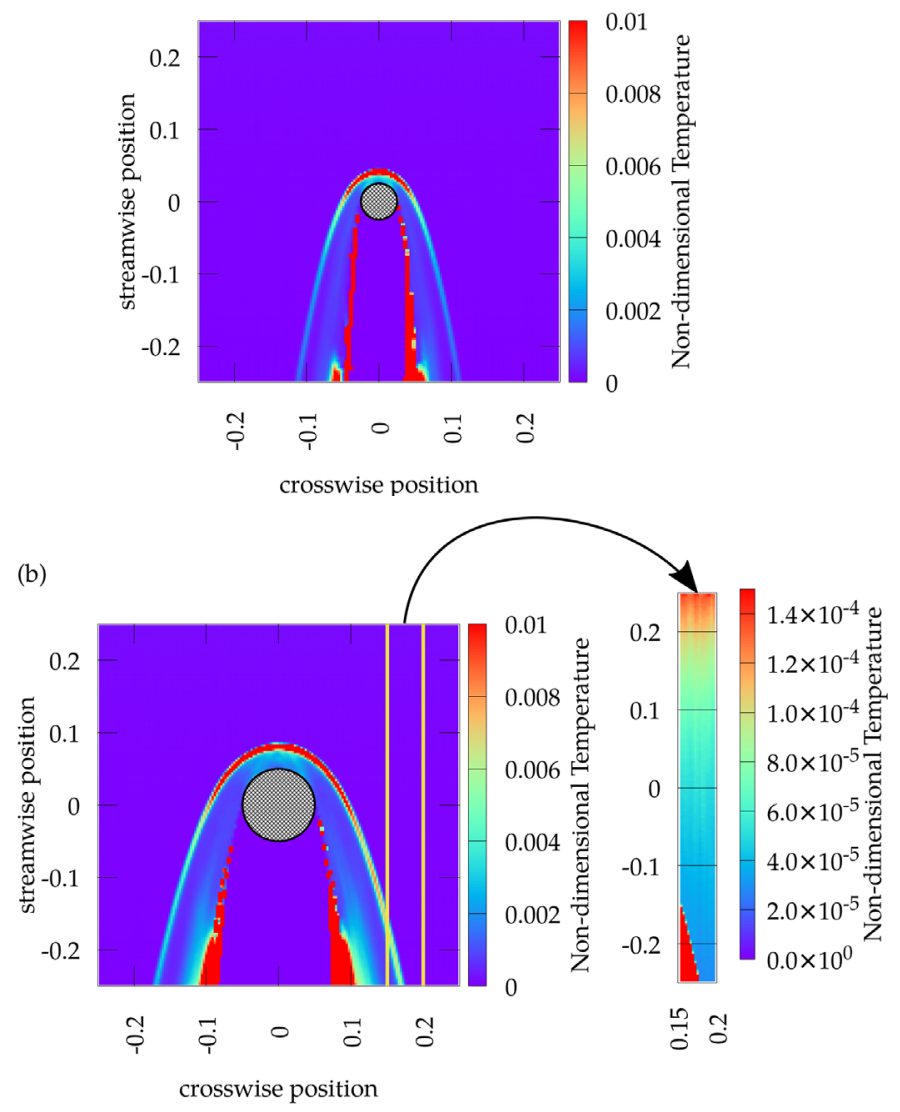

Figure 4: Granular temperature $\left(T / m_{a v} U_{\infty}^{2}\right)$ field, with $m_{a v}=\sum_{\alpha} m_{\alpha} / 2$; the parameter values are same as in Fig. 3 . The right panel in (b) displays a vertical-strip of the simulation box (enclosed by two yellow lines in the main panel) with a lower range of temperatures.

\subsection{Detachment Region and the Sonic Line}

To quantify the shock structure, the local Mach numbers (Ma) are calculated, see Fig. 5. An $M a=1$ iso-Mach line (i.e. the sonic line) is computed from the data which is zoomed as an inset panel in Fig. 5. The sonic-line (located just behind the shock-front) demarcates the subsonic and the supersonic regions of the flow - the shape of the sonicline looks much different from its molecular analog (see the schematic in Fig. 1). Comparing the Mach-number fields in Fig. 5 with the corresponding temperature-fields in Fig. 4, we find that the high temperature region is a region of low $M a$, representing a sub-sonic flow (since this region is clearly enveloped by the sonic-line). It has been verified that the high temperature region is a region of low velocities but of high velocity fluctuations (i.e. of higher values of granular temperature and sound speed) - this observation is in qualitative agreement with experimental results [6].

It must be pointed out that, for dissipative granular flows, the upstream Mach number $M a_{\infty}$ can be quite misleading. Due to inelastic collisions, the temperature $(T)$ 

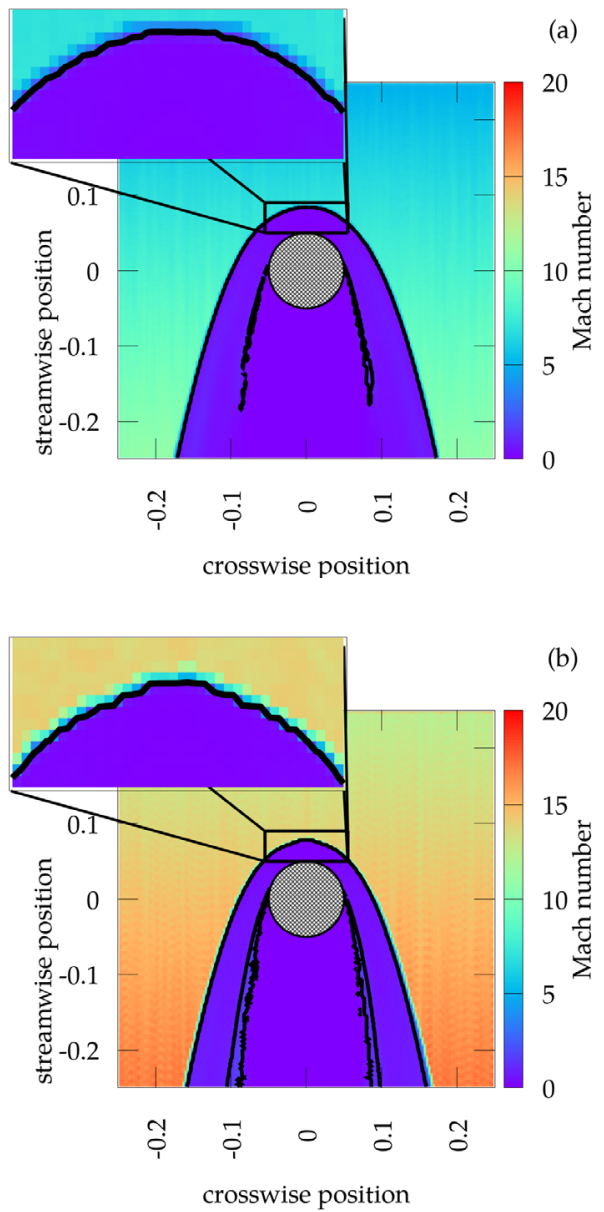

Figure 5: Local Mach number field for (a) $M a_{\infty}=5.9$ and (b) 13.7 with $D=100 d$. The inset is a magnified view of the detachment region, showing the sonic-line $(M a=1)$.

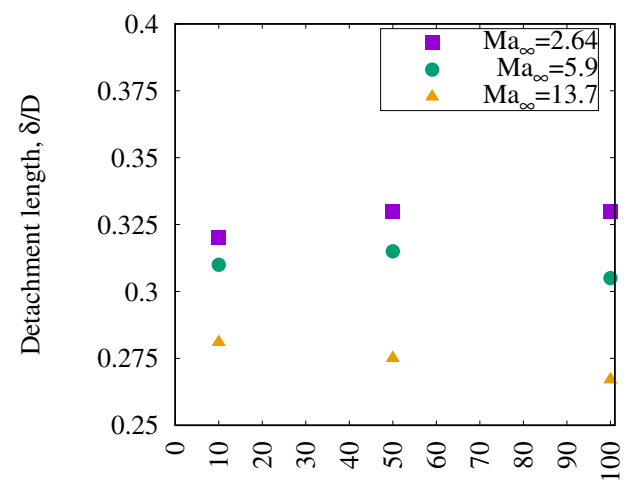

Obstacle diameter, D/d

Figure 6: Non-dimensional detachment length, $\delta / D$, plotted against obstacle diameter, $D / d$, for different $M a_{\infty}$.

(the right panel in Fig. 4b) and consequently the sound speed $(a)$ decays downstream - this in turn increases the "local" Mach number as the particles progress downstream. The upstream Mach number can thus be a misleading metric to characterize the "granular" shock since the obstacle would see a different (and in-fact larger) Mach number upstream from the point of collision with granular particles. A similar conclusion has recently been noted in the case of plane granular shock waves [5].

The non-dimensional detachment length, $\delta / D$, (see Fig. 1 for its definition) is plotted against $D / d$ in Fig. 6. It is seen that $\delta / D$ decreases with increasing $M a$ but remains nearly constant, with varying $D / d$, implying that the detachment length $(\delta)$ is a function of obstacle diameter $(D)$ for a given upstream Mach number, at least for the range of $M a_{\infty}$ considered in this study.

\section{Summary and Outlook}

DEM simulations of quasi-two dimensional dilute flows of bi-disperse spherical granular particles past a fixed cylindrical object were carried out. A high temperature region is observed in the "subsonic" detachment region, between the bluff body and the bow-shock front. It is found that inelastic collisions result in a decay of the temperature field along the flow direction, resulting in varying downstream Mach numbers (greater than $M a_{\infty}$ ). The detachment length $(\delta)$ is found to be a function of obstacle diameter $(D)$ for the range of $M a_{\infty}$ studied. The future work should focus on a better understanding of the bow-shock and the shape of the sonic line by quantifying the effects of (i) the mean density, (ii) the molar-fraction of binary mixture, (iii) the material parameters and (iv) the upstream Mach number.

\section{Acknowledgement}

Nandu Gopan would like to gratefully acknowledge the Science and Engineering Research Board (SERB) for support under the National Post-Doctoral Fellowship (PDF/2015/001068).

\section{References}

[1] E.C. Rericha, C. Bizon, M.D. Shattuck, H.L. Swinney, Phys. Rev. Lett. 88, 014302 (2001)

[2] T. Faug, P. Childs, E. Wyburn, I. Einav, Phys. Fluids 27, 073304 (2015)

[3] X. Cui, J. Gray, J. Fluid Mech. 720, 314 (2013)

[4] C.R. Wassgren, J.A. Cordova, R. Zenit, A. Karion, Phys. Fluids 15, 3318 (2003)

[5] M.H.L. Reddy, M. Alam, J. Fluid Mech. 779, R2 (2015)

[6] Y. Amarouchene, J.F. Boudet, H. Kellay, Phys. Rev. Lett. 86, 4286 (2001)

[7] T. Atkinson, J. Butcher, M. Izard, R. Nedderman, Chem. Engg. Sci. 38, 91 (1983)

[8] P.A. Cundall, O.D.L. Strack, Géotechnique 29, 47 (1979)

[9] C. Kloss, C. Goniva, A. Hager, S. Amberger, S. Pirker, Prog. Comput. Fluid Dyn. 12, 140 (2012)

[10] S. Savage, J. Fluid Mech. 194, 457 (1988) 